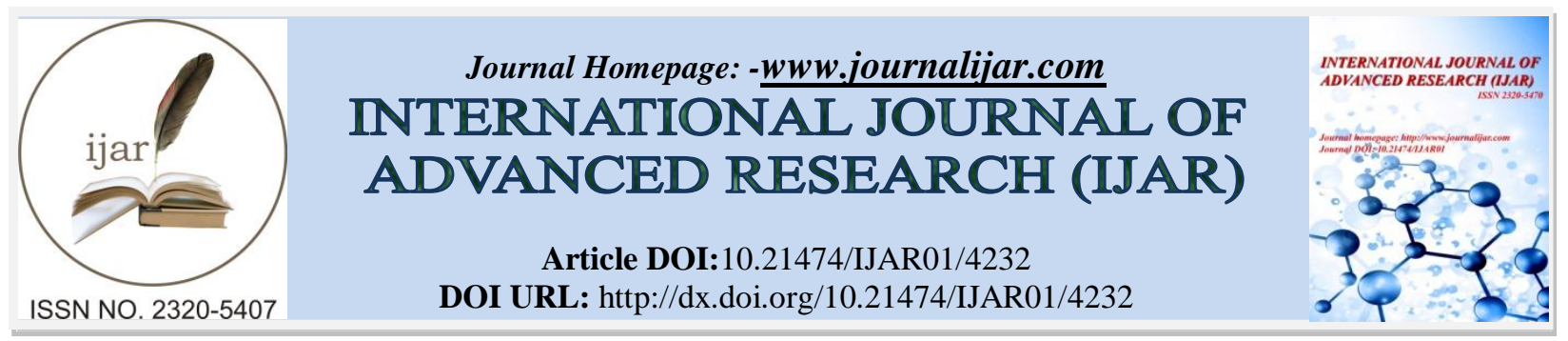

RESEARCH ARTICLE

\title{
EFFECT OF SENSOR NETWORKS IN NANO-ROBOTICS
}

\author{
Atul Ranjan Srivastava ${ }^{1}$, Amod Kumar Pandey ${ }^{2}$ and Kartik Goyal ${ }^{3}$ \\ 1. Assistant professor, Department of Electronics \& Communication Engineering, SRMSCET, Unnao, INDIA \\ 2. Research Scholar, University of Allahabad, Allahabad, INDIA \\ 3. Associate Member, IRED, Assistant professor, Department of Electronics \& Communication Engineering, \\ SRMSCET,Unnao, INDIA
}

\section{Manuscript Info}

Manuscript History

Received: 19 March 2017

Final Accepted: 22 April 2017

Published: May 2017

Key words:-

Sensor network, data transmission, molecular sorting rotor, locomotion, IC.

\section{Abstract}

This paper deals with the basic construction and applications of nanorobots in various fields. The important aspects of nanosensors and their functions are discussed in detail. Nanorobots are expected to revolutionize the medical industry, as they can treat at cellular level and make medical applications easy and effectivemicroscopic robots. Nanorobots raise all the important issues that must be addressed in NEMS like design, sensing, actuation, control, communications, power, and interfacing across spatial scales and between the organic/inorganic and biotic/abiotic realms. They can be also used for environmental monitoring purposes. Nanorobotics encompasses the design, fabrication and programming of robots with overall dimensions in the submicron range, and the manipulation of nanoscale objects with nanorobots.

Copy Right, IJAR, 2017,. All rights reserved.

\section{Introduction:-}

Nanorobots are Nano devices that are few micrometers $(0.1-10 \mu \mathrm{m})$ in size. The individual parts used to make these nanorobots are of nanoscale. They are mainly made up of carbon and Nanoelectromechanicalsystems (NEMS). They may be given a coating of diamond, diamond coat is given because it is the most inert and tough material ever known. Nanorobots are expected to revolutionize the medical industry, as they can treat at cellular level and make medical applications easy and effective. They can be also used for environmental monitoring purposes.

\section{Components of A Nanorobot:-}

The main functions of a nanorobot are to identify disease causing cells, destroying them and also to transport drugs to the affected area. So, they must have actuators to move, Sensors to sense, data transmitters to transmit data to the outside world and power supply. There are three main considerations scientists need to focus on when looking at nanorobots moving through the body navigation, powerand how the nanorobot will move through blood vessels

\section{Navigation:-}

External navigation systems use a variety of different methods to guide the nanorobot to the right location. One of these methods is to use ultrasonic signals to detect the nanorobot's location and direct it to the right destination. The nanorobot could emit pulses of ultrasonic signals, which can be detected using special equipment with ultrasonic sensors. 


\section{Powering the Nanorobot:-}

A nanorobot can be powered by using either external or internal power source. Nanorobots could get power directly from the bloodstream. A nanorobot with mounted electrodes could form a battery using the electrolytes found in blood. Another option is to create chemical reactions with blood to burn it for energy. The nanorobot would hold a small supply of chemicals that would become a fuel source when combined with blood. It might be possible to create batteries small enough to fit inside a nanorobot; they aren't generally seen as a viable power source. The problem is that batteries supply a relatively small amount of power related to their size and weight, so a very small battery would only provide a fraction of the power a nanorobot would need.

\section{Locomotion:-}

There are different kinds of actuators, such as electromagnetic, piezoelectric, electrostatic, and electro thermal, which may be utilized depending on the aim and the workspace where the actuator is to be applied. A set of fullerene structures was presented as Nano actuators. Nanorobots need a means of propulsion to get around the body.

\section{Sensor:-}

Through the use of nanowires, existing significant costs of energy demand for data transfer and circuit operation can be decreased by up to $60 \%$. CMOS-based sensors using nanowires as material for circuit assembly can achieve maximal efficiency for applications regarding chemical changes, enabling new medical applications. Sensors with suspended arrays of nanowires assembled into silicon circuits, decrease drastically self-heating and thermal coupling for CMOS functionality. Factors like low energy consumption and high-sensitivity are among some of the advantages of nanosensors. Nano sensor manufacturing array processes can use electro fluidic alignment to achieve integrated CMOS circuit assembly as multi element systems.

\section{Data transmission:-}

The application of devices and sensors implanted inside the human body to transmit data for a person health care can enable great advantages in continuous medical monitoring. It can also provide an innovative tool for accurate and in time prognosis of contagious diseases. Most recently, the use of RFID for data collecting and transmission was successfully tested for electroencephalograms. For communication in liquid workspaces, depending on the application, acoustic, light, RF, and chemical signals may be considered as possible choices for communication and data transmission.

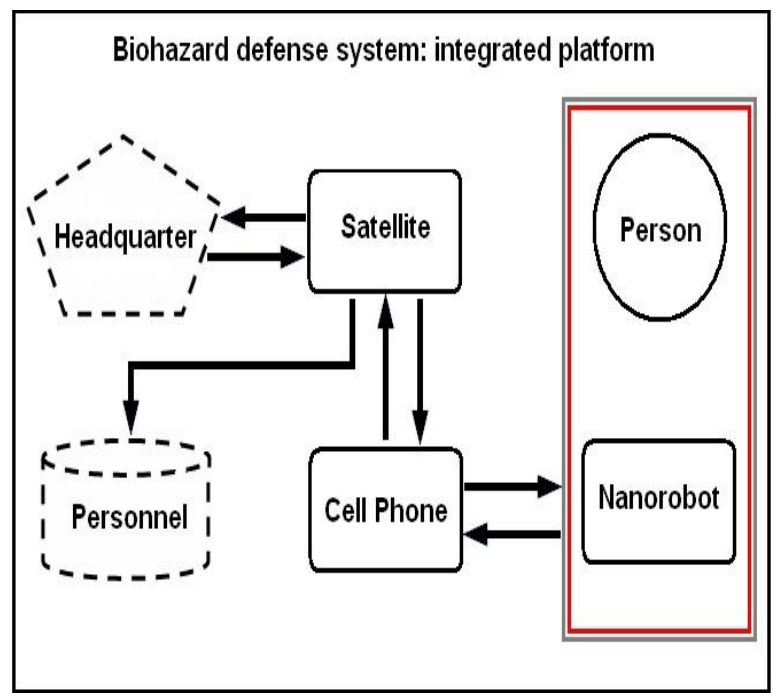

Fig1:-Biohazard defense system (Data transmission)

The application of ultra-high frequency satellite communications network can be successfully applied for nanorobot data transmission, using wireless phones for long distance communication. The cell phone PDA (personal digital assistant) system provides also the person's identification with respective position for the moment the nanorobot detected some virus protein profile. 
Nanorobot IC Layout: The main parameters used for the nanorobot architecture and control activation, as well as the required technology background that can advance manufacturing hardware for molecular machines, are described next. As a practical rule, the number of nanodevices to integrate into a nanorobot should be kept small to ensure that the hardware size is suitable for inside-body application.

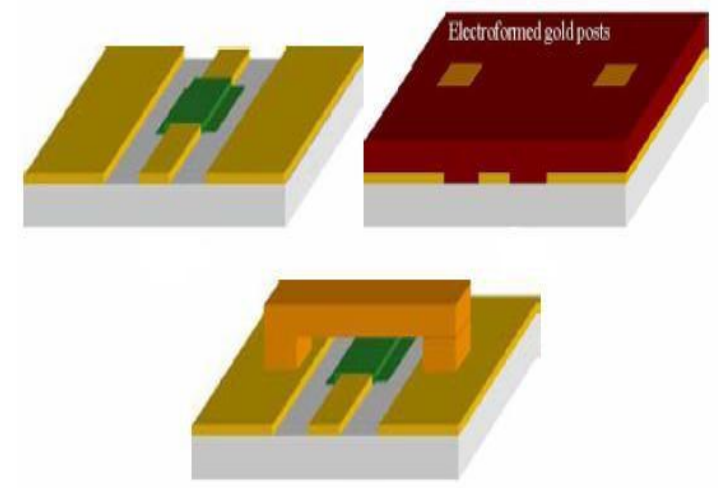

Fig2:- CMOS (Complementary metal-oxide semiconductor)

CMOS achieved 10nm sizes functionality they can be used as embedded nano device to build integrated sensors and actuator for nanorobots. RF-CMOS with wireless communication is a feasible way to interface with nanorobots for tracking, operation and diagnosis.

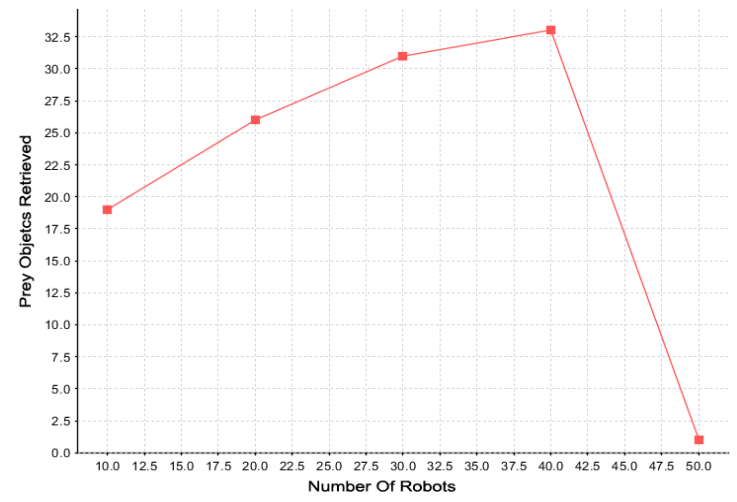

Fig3:-Undivided strategies.

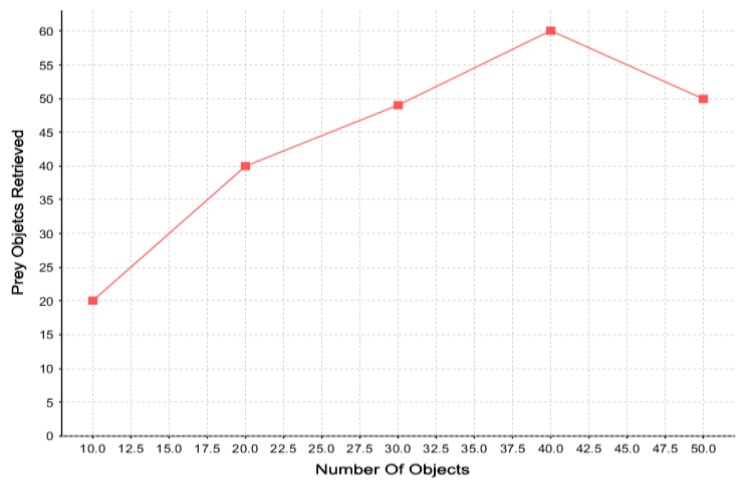

Fig4:-Divided strategies. 


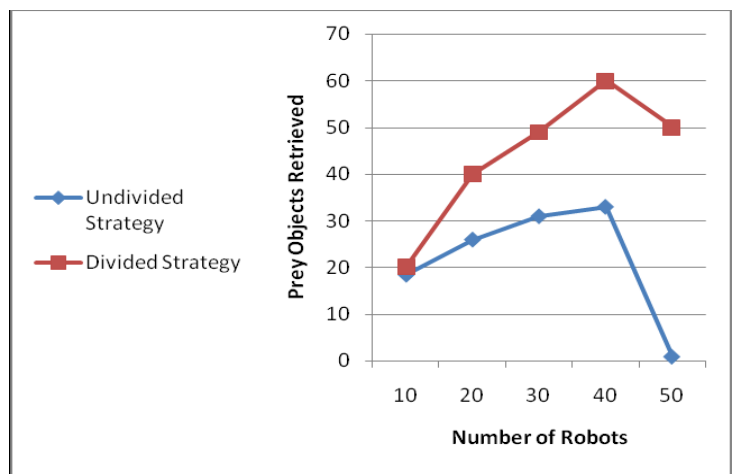

Fig5:-Performance of Undivided and devided strategies.

\section{NanorobotDesign:-}

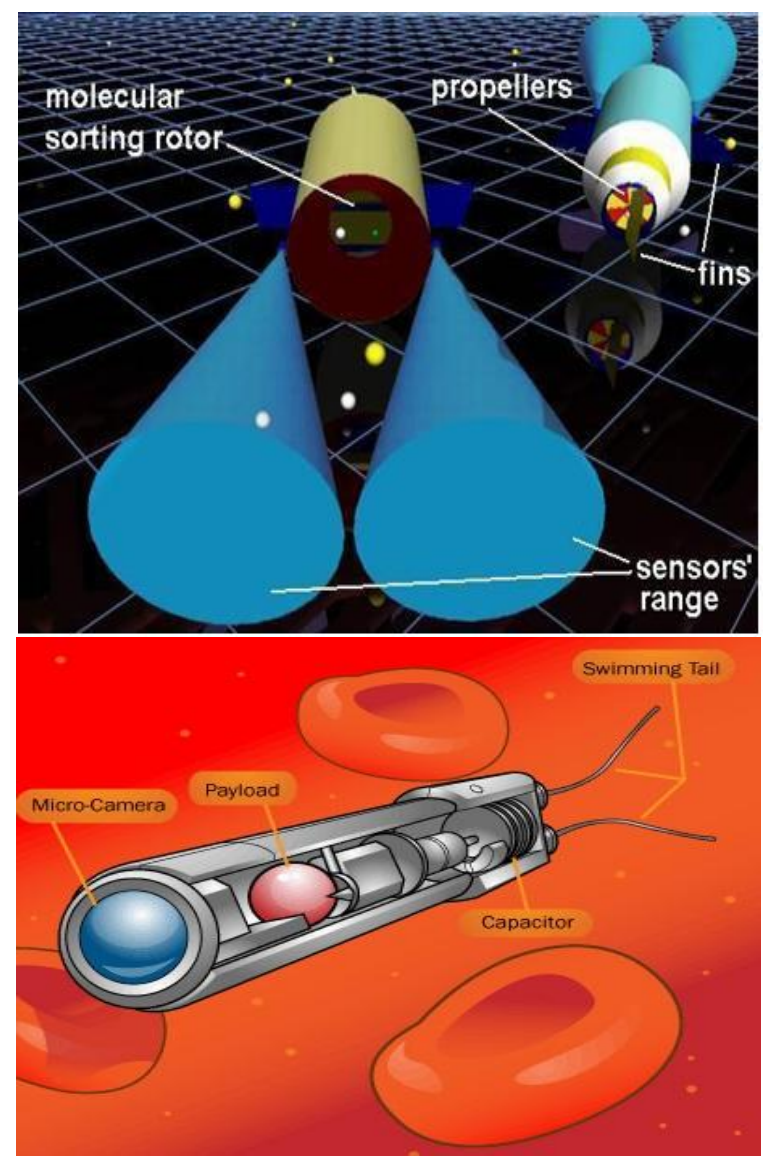

Fig 5:-Proposed models of nanorobots

\section{Conclusion:-}

In the future, nanorobots could revolutionize medicine. Doctors could treat everything from heart disease to cancer using tiny robots the size of bacteria, a scale much smaller than today's robots. Robots might work alone or in teams to eradicate disease and treat other conditions. Doctors would implant robots able to patrol a human's body, reacting to any problems that pop up. Unlike acute treatment, these robots would stay in the patient's body forever.

Another potential future application of nanorobot technology is to re-engineer our bodies to become resistant to disease, increase our strength or even improve our intelligence. Dr.Richard Thompson, a former professor of ethics, has written about the ethical implications of nanotechnology. He says the most important tool is communication, and 
that it's pivotal for communities, medical organizations and the government to talk about nanotechnology now, while the industry is still in its infancy.

\section{References:-}

1. Adriano Cavalcanti, BijanShirinzadeh, Mingjun Zhang and Luiz C. Kretly "Nanorobot Hardware Architecture for Medical Defense", 6 May 2008.

2. Arai, F., Liu, P., Dong, L., Fukuda, T., 2004, "Field emission property characterization of individual carbon nanotubes through nanorobotic manipulations and its applications," Proceedings of the IEEE International Conference on Robotics and Automation, New Orleans

3. Arai, F., Nakajima, M., Dong, L., Fukuda, T., 2003a, "Pico-Newton order force measurement using a calibrated carbon nanotube probe by electromechanical resonance

4. Adriano cavalcanti, BijanShirinzadeh, Robert A FreitasJr and Tad Hogg 2008 "Nanorobotarchitecturefor medical target identification" IOP Publishing.

5. Freitas R A Jr 1999 "Nanomedicinevol I Basic Capabilities" Landes Bioscience http://www.nanomedicine.com.

6. Cavalcanti A 2003 "Assembly automation with evolutionary nanorobots and sensor (based control applied to nanomedicine" IEEE Trans. Nanotechnoly.

7. Montemagno C D and Bachand G D 1999 "Constructing nanomechanical devices powered by biomolecular motors" Nanotechnology $10225(231$.

8. Cavalcanti A, Shirinzadeh B, Freitas R A Jr and Kretly L C 2007 "Medical nanorobot architecture based on nanobioelectronics" Recent Patents on Nanotechnologyvol 1 (Pennington, NJ, USA: Bentham Science Publishers Ltd.) pp 1(10.C. J. Kaufman, Rocky Mountain Research Lab., Boulder, CO, private communication, May 1995.

9. .Montemagno C D and Bachand G D 1999 Constructing nanomechanical devices powered by biomolecular motors Nanotechnology 10 225-31.

10. Cavalcanti A, Shirinzadeh B, Freitas R A Jr and Kretly L C 2007 Medical nanorobot architecture based on nanobio electronics Recent Patents on Nanotechnology vol 1 (Pennington, NJ, USA: Bentham Science Publishers Ltd.) pp 1-10.

11. Mathieu J B, Beaudoin G and Martel S 2006 Method of propulsion of a ferromagnetic core in the cardiovascular system through magnetic gradients generated by an MRI system IEEE Trans. Biomed. Eng. 53 292-299. 